Conclusions Nasobiliary drainage is a safe endoscopic technique that improves symptomatic cholestasis. The drainage catheters are well tolerated by patients with an acceptable sideeffect profile.

\section{PTU-17 A COMPARISON BETWEEN ENDOSCOPIC AND RADIOLOGICALLY PLACED DUODENAL STENTS IN MALIGNANT GASTRIC OUTLET OBSTRUCTION}

George Hiner*, Wafaa Ahmed, Anum Javed, Panagiotis Vlavianos, Christopher Wadsworth, Natalie Phillips, Devinder Bansi, Shwan Karim. Imperial College Healthcare NHS Trust, London, UK

\subsection{6/gutjnl-2021-BSG.90}

Introduction Duodenal stenting is an established palliative therapy in malignant gastric outflow obstruction. This can be carried out either endoscopically or via interventional radiology (IR) depending on clinician experience and resources. We sought to assess the efficacy and safety of these two methods in our centre.

Methods Patients who underwent duodenal stenting via endoscopy or IR from July 2014 to November 2020 were retrospectively identified. Unique patient identification numbers were cross referenced with the endoscopy database and electronic record. Patient demographics, procedure indication, success rates and complication rates were recorded. Technical success was defined as safe insertion of the duodenal stent across the stricture. Clinical success was defined as relief of symptoms related to gastric outlet obstruction (Grades; 0 fail, 1 - liquid diet, 2 - soft diet, 3 - solid diet). Statistical analysis was performed using Fisher's Test with $\mathrm{p}$ value of $<$ 0.05 being significant.

Results 92 patients underwent 114 procedures. Stenting was carried out via endoscopy in 78 patients (103 stents, age median 69 (32-90)), with 17 patients having stents inserted via IR (27 stents, age median 63 (46-88)). The median survival for both groups was 167.9 days following their first stent insertion.

In the endoscopic intervention group, 63 had gastric outlet obstruction secondary to an upper GI cancer and 15 had metastatic disease from a non-upper GI primary. 4 of the patients had a previous Whipple's procedure and 1 had a gastrojejunostomy. In the IR group, 16 had an upper GI cancer, 1 had metastatic colorectal cancer. 1 of these patients had a previous gastrojejunostomy.

There were no immediate serious post procedure complications in either group. 2 patients in the endoscopic group had early stent migration (within 30 days of insertion) and 1 patient's stent did not fully open and required balloon dilatation. Similarly, in the IR group, 1 patient's stent did not fully open requiring balloon dilatation of the stent and an additional stent insertion.

In the endoscopic group, technical success was achieved in $101(98.1 \%)$ stents compared to $25(92.6 \%)$ in IR ( $\mathrm{p}=$ 0.19 ). A patient with previous gastrojejunostomy underwent a combined endoscopic and radiological procedure unsuccessfully. In the endoscopic group, 1 stent was inadvertently deployed through an existing biliary metal stent. On further analysis, we observed that more than one stent was inserted more frequently in the IR group, 13 of 20 procedures (65\%), compared to 10 of $93(10.8 \%)$ endoscopic procedures $(p=$ $0.003)$. Tumour ingrowth and stent obstruction occurred in 12
$(12.8 \%)$ procedures in the endoscopy group vs $2(10 \%)$ in IR group within 6 months from insertion.

Clinical success was achieved in endoscopic stenting in 95 (92.4\%) of the stents vs $23(85.2 \%)$ through IR $(p=0.27)$. Further analysis comparing the type of diet patients could tolerate was available for 51 (out of 77) of the patients in the endoscopic group and for all 17 patients in the IR group. In the endoscopically inserted group, 22 patients tolerated solid diet (43.1\%), 18 tolerated soft diet $(35.3 \%)$ and 6 tolerated liquid diet (11.8\%) vs $3(17.6 \%), 10(58.8 \%)$ and $2(11.8 \%)$ in the IR group respectively.

Conclusion In malignant gastric outlet obstruction, duodenal stents inserted endoscopically or radiologically, can be performed safely and allow patients to live with improved symptoms for an extended period of time. In our cohort, it appears that radiologically, 2 overlapping stents are more frequently required to achieve the desired effect. We suspect this is because of endoscopic direct visualisation of the obstruction aids stent position on insertion.

\section{PTU-18 ENDOSCOPY PROCEDURE VOLUME AMONG THE UK ENDOSCOPIST WORKFORCE: ANALYSIS USING THE NATIONAL ENDOSCOPY DATABASE (NED)}

${ }^{1}$ David Beaton*, ${ }^{2}$ Raph Broughton, ${ }^{3}$ Peter Rogers, ${ }^{4}$ Linda Sharp, ${ }^{5}$ Mo Thoufeeq, ${ }^{6}$ Nigel Trudgill, ${ }^{7}$ Brian Nicholson, ${ }^{1,4}$ Matt Rutter. ${ }^{1}$ North Tees and Hartlepool NHS Foundation Trust; ${ }^{2}$ Royal College of Physicians, London; ${ }^{3}$ WebLogik.co.uk; ${ }^{4}$ Population Health Sciences, Newcastle University; ${ }^{5}$ Sheffield Teaching Hospitals NHS Trust; ${ }^{6}$ Sandwell and West Birmingham NHS Trust; ${ }^{7}$ Department of Healthcare Sciences, Oxford University

\subsection{6/gutjnl-2021-BSG.91}

Introduction The British Society of Gastroenterology recommends endoscopists perform a minimum number of endoscopic procedures annually to maintain competence: OGD \& colonoscopy 100, ERCP 75.

This study aimed to use the National Endoscopy Database (NED) to identify the proportion of the UK endoscopist workforce performing fewer than recommended annual procedures, and their contribution to overall activity.

Methods NED records endoscopy procedural data in real time; over $80 \%$ of UK endoscopy sites now upload to NED.

All procedures recorded from 1st March 2019 to 29th Feb 2020 were analysed. Endoscopy sites not uploading throughout the entire study period were excluded. Trainees were excluded, as they are not subject to the recommended minimum procedures, as were endoscopists solely uploading from independent sector (IS).

Median annual procedures included those performed in the NHS and IS and were computed overall and for doctor and nurse endoscopists (NE) separately. The percentage of endoscopists undertaking less than the recommended minimums, and the percentage of all procedures done by these endoscopists were calculated.

Result 187 NHS sites were included in the analysis, covering 1,230,822 procedures. The workforce comprised 3377 doctors $(87.5 \%)$ and $484 \mathrm{NE}(12.5 \%)$. Doctors performed 924,976 (75.2\%) procedures and NE 305,855 (24.8\%). Analysis of individual procedures is shown in Table 1.

Conclusion Nurse endoscopists perform substantially more procedures than doctors (median per endoscopist) and perform a quarter of all UK endoscopy.

Around half of UK endoscopists do not reach the minimum annual number of procedures for OGDs, colonoscopies or 